\title{
XMM-Newton EPIC and RGS observations of LMC X-3
}

\author{
K. Wu ${ }^{1,2}$, R. Soria ${ }^{1}$, M. J. Page ${ }^{1}$, I. Sakelliou ${ }^{1}$, S. M. Kahn ${ }^{3}$, and C. P. de Vries ${ }^{4}$ \\ 1 Mullard Space Science Laboratory, University College London, Holmbury St Mary, Dorking, RH5 6NT, UK \\ 2 Research Centre for Theoretical Astrophysics, School of Physics, University of Sydney, NSW 2006, Australia \\ 3 Columbia Astrophysics Laboratory, Columbia University, 550 West 120th Street, New York, NY 10027, USA \\ ${ }^{4}$ Space Research Organization of The Netherlands, Sorbonnelaan 2, 3584 CA Utrecht, The Netherlands
}

Received 2 October 2000 / Accepted 10 November 2000

\begin{abstract}
We report the results of preliminary analysis of the XMM-Newton EPIC and RGS observations of the candidate black-hole binary LMC X-3 between February and June 2000. The observations covered both the soft and the hard $\mathrm{X}$-ray spectral states. The hard-state spectra were dominated by a power-law component with a photon index $\Gamma \approx 1.9$. The soft-state spectra consisted of a thermal component with a multi-colour disk temperature $T_{\text {in }}$ of $\sim 0.9 \mathrm{keV}$ and a power-law tail with $\Gamma \approx 2.7$. The model in which the X-rays from LMC X-3 in the high-soft state are powered by a strong stellar wind from a massive companion is not supported by the small line-of-sight absorption $\left(n_{\mathrm{H}} \lesssim 10^{21} \mathrm{~cm}^{-2}\right)$ deduced from the RGS data. The transition from the soft to the hard state appears to be a continuous process associated with the changes in the mass-transfer rate.
\end{abstract}

Key words. accretion, accretion disks - black hole physics - binaries: general - stars: early type - stars: winds, outflows - X-rays: general

\section{Introduction}

LMC X-3 (Leong et al. 1971) is a persistent X-ray source in the Large Magellanic Cloud (LMC). It has an orbital period of $1.70 \mathrm{~d}$, and a mass function $\simeq 2.3 M_{\odot}$ (Cowley et al. 1983). The optical brightness of $V \sim 17$ indicates that the system has a massive companion (van Paradijs et al. 1987). The companion is often classified as a B3 V star (Cowley et al. 1983), although a B5 IV spectral type has also been suggested (Soria et al. 2001). The non-detection of eclipses in the X-ray curve implies that the orbital inclination of the system is $\lesssim 70^{\circ}$ (Cowley et al. 1983). The inferred mass of the compact star is $\gtrsim 7 M_{\odot}$ (Paczynski 1983), thus establishing that the system is a black-hole candidate (BHC).

Most BHCs show soft and hard X-ray spectral states. Their X-ray spectrum in the soft state generally consists of both a thermal and a power-law component. The thermal component can be fitted by a blackbody spectrum with a temperature $\sim 1 \mathrm{keV}$, and it is interpreted as thermal emission from the inner accretion disk. The power-law component is believed to be comptonised emission from a disk corona (Sunyaev \& Titarchuk 1980) or from the high-speed infalling plasma near the black-hole event horizon (Titarchuk \& Zannias 1998). The photon index $\Gamma$ of the power law is $\approx 2.5-4$. In the hard state, the thermal

Send offprint requests to: $\mathrm{K}$. Wu component is insignificant. The spectrum is dominated by a flat $(1.5 \lesssim \Gamma \lesssim 2)$, extended power-law component.

LMC X-3 is found in the soft state most of the time. LMC X-1, another high-mass BHC in the LMC, has been seen in the soft state only. In contrast, Cyg X-1, the highmass BHC in our Galaxy, tends to be in the hard state for the majority of the time. Such preference for either the hard or the soft state is not evident in the low-mass transient BHCs (e.g. GRO J1655-40). It is still unclear how mass transfer occurs in LMC X-3: whether via Roche-lobe overflow or capture of the stellar wind from the companion. Given the fact that a B3 V star is unable to drive a strong stellar wind or to fill its Roche lobe if it is in a binary system with a 1.7-d orbital period, it is difficult to explain the observed X-ray luminosity of the system.

In this letter, we report on the $X M M$-Newton observations of LMC X-3 between 2000 February and June. The system was in an unusual hard state in April, and our observations span the transition from the soft state to the hard state and then back to a softer state. Making use of these new X-ray spectral data, we attempt to shed light on the question of mass transfer in the system (see also Soria et al. 2001). 
Table 1. XMM-Newton EPIC observation log

\begin{tabular}{llll}
\hline Revolution/ID & Instrument & Exposure $^{a}$ & Mode $^{b}$ \\
\hline \hline Rev0028/201 & PN & $08.1 \mathrm{ks}$ & small window \\
Rev0028/301 & PN & $0.3 \mathrm{ks}$ & full PN \\
& MOS1 & $08.9 \mathrm{ks}$ & partial RFS \\
Rev0030/501 & MOS1 & $05.9 \mathrm{ks}$ & partial W5 \\
Rev0041/101 & PN & $0.7 \mathrm{ks}$ & full PN \\
Rev0041/401 & MOS1 & $03.5 \mathrm{ks}$ & partial W2 \\
& MOS1 & $03.6 \mathrm{ks}$ & partial W5 \\
& MOS1 & $03.4 \mathrm{ks}$ & partial W4 \\
Rev0045/101 & PN & $01.6 \mathrm{ks}$ & full PN \\
Rev0045/301 & MOS1 & $06.6 \mathrm{ks}$ & partial W3 \\
Rev0066/101 & PN & $26.6 \mathrm{ks}$ & small window \\
Rev0092/201 & PN & $24.2 \mathrm{ks}$ & small window \\
& MOS1 & $20.3 \mathrm{ks}$ & partial W4 \\
\hline
\end{tabular}

${ }^{a}$ For the PN small window mode, the live time was $71 \%$ of the exposure time listed here; for the MOS RFS mode the live time was $6.9 \%$ of the exposure time.

${ }^{b}$ See XMM Remote Proposal Submission Software Users' Manual.

${ }^{c}$ Only the first $8.3 \mathrm{ks}$ are useful.

\section{Observations}

\subsection{Technical details}

LMC X-3 was observed with the European Photon Imaging Camera (EPIC; Turner et al. 2001), the Reflection Grating Spectrograph (RGS; Brinkman et al. 2001) and the Optical Monitor (OM; Mason et al. 2001) on board XMM-Newton, during the Performance Verification Phase between 2000 February and June. Here we present part of the EPIC and RGS observations. The OM observations are presented in Soria et al. (2001). The logs of the EPIC-MOS1/PN and RGS observations used for this study are shown in Tables 1 and 2 respectively. The EPIC-MOS2 observations are not presented, as a reliable response matrix is not yet available. The exposure times listed in Table 1 take into account the counting mode interruptions. In some cases, the fraction of live time during which photons were actually collected is significantly smaller than the total exposure time.

All EPIC exposures were taken with the "medium" filter; Some of the data suffer from pile-up when the source was observed in the bright state (before Rev0066). MOS exposures taken in the "full window" mode are worst affected, and so are excluded from the analysis. "Partial window" MOS exposures and "full window" PN exposures are, however, less affected. In our analysis, we have removed the central pixels (which are most affected by pileup) from the extraction regions, for the partial MOS and full PN exposures that are affected. The corresponding normalisations of the fitted spectral models for these observations are therefore smaller than the true values, and
Table 2. XMM-Newton RGS observation log

\begin{tabular}{llll}
\hline Revolution/ID & Instrument $^{a}$ & Exposure & count rate $^{b}$ \\
\hline \hline Rev0028/201 & RGS2 (s1) & $17.6 \mathrm{ks}$ & $4.79 \pm 0.02$ \\
Rev0030/501 & RGS2 (tot) & $12.1 \mathrm{ks}$ & $4.52 \pm 0.03$ \\
Rev0045/101 & RGS1 (s1) & $06.4 \mathrm{ks}$ & $3.59 \pm 0.03$ \\
& RGS2 (s1) & $06.4 \mathrm{ks}$ & $3.56 \pm 0.03$ \\
Rev0045/201 & RGS1 (s1) & $06.7 \mathrm{ks}$ & $3.62 \pm 0.02$ \\
& RGS2 (s1) & $06.8 \mathrm{ks}$ & $3.57 \pm 0.02$ \\
Rev0045/301 & RGS1 (s1) & $03.0 \mathrm{ks}$ & $3.66 \pm 0.04$ \\
& RGS1 (s3) & $03.0 \mathrm{ks}$ & $3.57 \pm 0.04$ \\
& RGS1 (s5) & $03.0 \mathrm{ks}$ & $3.40 \pm 0.04$ \\
& RGS2 (s2) & $00.3 \mathrm{ks}$ & $3.63 \pm 0.17$ \\
& RGS2 (s4) & $03.0 \mathrm{ks}$ & $3.26 \pm 0.04$ \\
& RGS2 (s6) & $00.3 \mathrm{ks}$ & $3.18 \pm 0.17$ \\
Rev0066/101 & RGS1 (s7) & $44.3 \mathrm{ks}$ & $0.0154 \pm 0.0012$ \\
& RGS2 (s8) & $44.3 \mathrm{ks}$ & $0.0153 \pm 0.0012$ \\
Rev0092/101 & RGS1 (s2) & $51.9 \mathrm{ks}$ & $1.32 \pm 0.01$ \\
& RGS2 (s2) & $51.9 \mathrm{ks}$ & $1.53 \pm 0.01$ \\
Rev0092/201 & RGS1 (s4) & $25.6 \mathrm{ks}$ & $1.56 \pm 0.01$ \\
& RGS2 (s5) & $25.6 \mathrm{ks}$ & $1.34 \pm 0.01$ \\
\hline
\end{tabular}

a The labels "s\#" identifies multiple exposures during the same revolution; for more details see the Preferred Observation Sequence files of each revolution.

${ }^{b}$ In photons $\mathrm{s}^{-1}$.

they are put in brackets in Table 3 . We are aware that the photon index of the power-law component is affected by pile-up. For the Rev0028, 0030, 0041 and 0045 observations, we estimate that the fitted values are $\approx 0.15-0.20$ smaller (harder) than the real value. The "small window" PN exposures are not significantly affected by pile up, and they are more reliable for the determination of the powerlaw photon indices.

The data were processed using the September 2000 release of the SAS, except for the PN small window mode data, which were processed with the October 2000 SAS release. All spectral fits presented here were performed with the most up-to-date response matrices available at the end of October 2000 (mos1_medium_all_qe17_rmf3_tel5_15.rsp for EPIC-MOS1 and epn_fs20_sY9_medium.rmf for EPIC$\mathrm{PN})$. We are aware that the PN response matrix assumes a nominal event threshold of $20 \mathrm{adu}$, whereas a threshold of 23 adu was used in the Rev0028 and Rev0041 observations. This may have a small effect on the low-energy end of the spectrum and make the determination of the column density from the early PN data less reliable. We used the RGS spectra to obtain a better determination of the column density. 

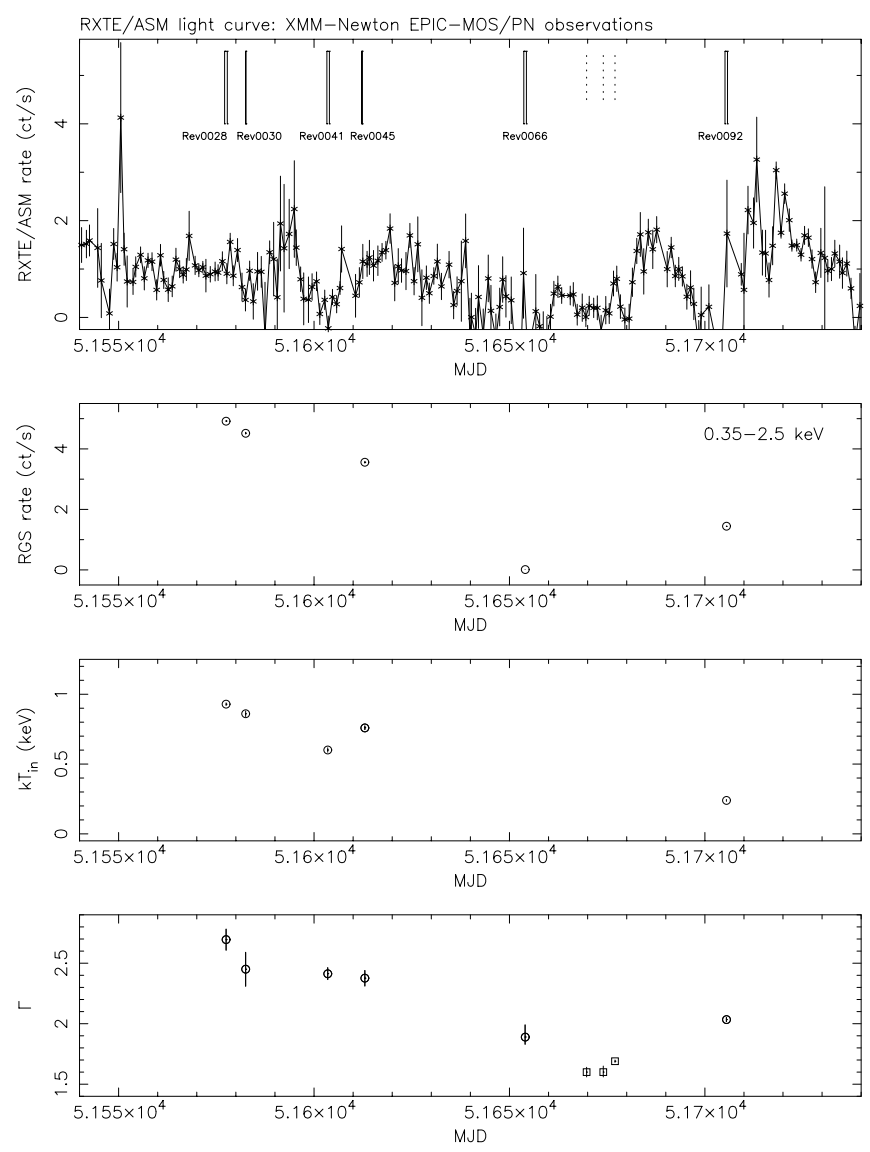

Fig. 1. (First panel from the top): the XMM-Newton EPIC observations of LMC X-3, marked by solid vertical lines, are shown and compared with the $R X T E / A S M$ light curve. The three $R X T E / \mathrm{PCA}$ observations that confirmed the hard state of the system (Boyd et al. 2000) are marked by dotted vertical lines. (Second panel): the $0.35-2.5 \mathrm{keV}$ light curve from the RGS observations. (Third panel): the evolution of the fit temperature of the thermal component in the EPIC-PN and MOS spectra. The open circles are the means of the fit diskblackbody temperatures $T_{\text {in }}$ error-weighted for the observations during the same revolution. (Fourth panel): the evolution of the photon index $\Gamma$ of the power-law tail. The data points (open circles) are error-weighted means. The open squares are the photon indices obtained by Boyd et al. (2000) from the three $R X T E / \mathrm{PCA}$ observations on May 5.76, 10.01 and 13.94 UT; a model with a single power law was used

\subsection{The 2000 April low-hard state}

The X-ray luminosity of LMC X-3 appeared to be declining during our February-March observations, with the $R X T E / A S M ~ 2-20 \mathrm{keV}$ count rate generally below $3 \mathrm{ct} \mathrm{s}^{-1}$ (Fig. 1). The Rev0066/101 observation (2000 April 19) was carried out around the middle of a faint-hard state, where the RXTE/ASM count rate was consistent with zero. The RXTE/PCA data obtained on May 5.76 and 10.01 UT showed power-law spectra with a photon index $1.60 \pm 0.05$ and a soft $(2-10 \mathrm{keV})$ X-ray flux of $\approx 5-910^{36} \mathrm{erg} \mathrm{s}^{-1}$ at $50 \mathrm{kpc}$ (Boyd \& Smale 2000; Boyd et al. 2000). The system seemed to be in the process of returning to the soft state in the Rev0092 (June 9-10) observations.

\subsection{EPIC MOS and PN data}

We consider a conventional model consisting of an absorbed multi-colour disk blackbody plus a power law, wabs*Zvfeabs*(diskbb+powerlaw) in XSPEC, to fit the EPIC data. As the fitting process is limited by the reliability of the response matrices currently available, more sophisticated models or the inclusion of additional features (such as lines) are not warranted. The generic models used here are therefore sufficient for this preliminary analysis.

For the MOS observations, we consider only the channels in the $0.3-8.0 \mathrm{keV}$ energy range; for the PN observations we extend the energy range to $0.3-12.0 \mathrm{keV}$. The data are binned with the FTOOLS task grppha, such that the number of counts in each group of channels is larger than 40. This improves the signal-to-noise ratio for energies $\gtrsim 6 \mathrm{keV}$, while it does not affect low-energy channels. We have also checked that different binning criteria give consistent results. No systematic error has been added to the data.

We consider the line-of-sight Galactic absorption toward LMC X-3 to be $3.210^{20} \mathrm{~cm}^{-2}$ following Wilms et al. (2000), and use it as the value for the (fixed) first absorption component. The second absorption component is then the intrinsic photoelectric absorption within the binary system and the LMC. We also fix both the iron abundance and the total metal abundance to be 0.4 times the solar values, to account for the lower metallicity of the LMC (Caputo et al. 1999). The best fit parameters are shown in Table 3. Figures 2-4 show the EPIC-PN spectra from the Rev0028/201, Rev0066/101 and Rev0092/201 observations respectively (all taken in small window mode, not affected by pile-up).

The reduced $\chi_{\nu}^{2}$ are about one in most fits. The large $\chi_{\nu}^{2}$ in some fits (e.g. the MOS1 observations in Rev0092/201) are probably due to the uncertain response and effective-area calibrations. In particular, the feature seen at $\approx 0.5 \mathrm{keV}$ in the PN spectra (Figs. 2-4) is due to uncertainties in the charge transfer efficiency correction for the small window mode. Values of $\chi_{\nu}^{2} \leq 1.05$ are obtained when the $0.4-0.6 \mathrm{keV}$ energy range is excluded from the fit.

\subsection{RGS data}

We consider the RGS data to search for possible emission lines, and to constrain the value of the column density. The RGS spectra are fitted with the same model used in the analysis of the PN data. As the RGS spectra cover only the $0.35-2.5 \mathrm{keV}$ energy range, the thermal and the power-law components cannot be constrained simultaneously. We therefore fix $\Gamma$ to the values determined from 
Table 3. Fits to the EPIC data: absorbed disk blackbody and power law

\begin{tabular}{|c|c|c|c|c|c|c|c|}
\hline Observation & & $n_{\mathrm{H}}^{a}$ & $T_{\text {in }}$ & $A_{\text {disk }}$ & $\Gamma$ & $A_{\mathrm{pl}}$ & $\chi_{\nu}^{2}(\mathrm{dof})$ \\
\hline Rev0028/201 & $\mathrm{PN}$ & $15.65_{-1.96}^{+2.10} 10^{20}$ & $0.93_{-0.01}^{+0.01}$ & $4.32_{-0.25}^{+0.24}$ & $2.71_{-0.08}^{+0.09}$ & $8.92_{-0.79}^{+0.83} 10^{-3}$ & $1.31(784)$ \\
\hline \multirow[t]{2}{*}{ Rev0028/301 } & $\mathrm{PN}$ & $4.82_{-4.82}^{+9.34} \quad 10^{20}$ & $0.93_{-0.05}^{+0.04}$ & $\left(6.26_{-1.15}^{+1.38}\right)$ & $2.45_{-0.44}^{+0.53}$ & $\left(\begin{array}{ll}6.04_{-3.58}^{+4.13} & \left.10^{-3}\right)\end{array}\right.$ & $1.02(192)$ \\
\hline & MOS1 (s7) & $17.98_{-8.12}^{+8.62} \quad 10^{20}$ & $0.88_{-0.06}^{+0.06}$ & $\left(0.65_{-0.15}^{+0.24}\right)$ & $2.53_{-0.35}^{+0.41}$ & $\left(1.55_{-0.50}^{+0.46} \quad 10^{-3}\right)$ & $0.75(170)$ \\
\hline Rev0030/501 & MOS1 (s7) & $13.00_{-3.53}^{+3.48} \quad 10^{20}$ & $0.86_{-0.02}^{+0.02}$ & $\left(6.10_{-0.65}^{+0.74}\right.$ & $2.45_{-0.14}^{+0.14}$ & $\left(\begin{array}{ll}9.60_{-1.70}^{+1.65} & \left.10^{-3}\right)\end{array}\right.$ & $1.28(282)$ \\
\hline Rev0041/101 & $\mathrm{PN}$ & $\begin{array}{ll}13.66_{-6.94}^{+6.17} & 10^{20}\end{array}$ & $0.59_{-0.06}^{+0.07}$ & $\left(10.91_{-5.17}^{+4.13}\right)$ & $2.57_{-0.27}^{+0.22}$ & $\left(\begin{array}{ll}10.60_{-3.43}^{+3.05} & 10^{-3}\end{array}\right)$ & $1.01(259)$ \\
\hline \multirow[t]{3}{*}{ Rev0041/401 } & $\operatorname{MOS} 1(\mathrm{~s} 3)$ & $15.08_{-2.69}^{+2.59} 10^{20}$ & $0.62_{-0.03}^{+0.04}$ & $16.80_{-4.36}^{+5.25}$ & $2.47_{-0.08}^{+0.08}$ & $2.97_{-0.33}^{+0.32} 10^{-2}$ & $1.12(260)$ \\
\hline & MOS1 (s5) & $10.31_{-2.63}^{+2.56} 10^{20}$ & $0.64_{-0.02}^{+0.03}$ & $16.65_{-3.37}^{+3.85}$ & $2.33_{-0.09}^{+0.08}$ & $2.20_{-0.28}^{+0.27} 10^{-2}$ & $1.22(258)$ \\
\hline & MOS1 (s7) & $13.28_{-2.80}^{+2.75} \quad 10^{20}$ & $0.58_{-0.02}^{+0.02}$ & $30.38_{-6.12}^{+6.62}$ & $2.41_{-0.09}^{+0.09}$ & $2.80_{-0.38}^{+0.37} 10^{-2}$ & $1.25(258)$ \\
\hline Rev0045/101 & $\mathrm{PN}$ & $6.15_{-3.65}^{+3.65} \quad 10^{20}$ & $0.76_{-0.02}^{+0.03}$ & $\left(9.00_{-1.33}^{+1.46}\right)$ & $2.32_{-0.17}^{+0.14}$ & $\left(8.14_{-2.04}^{+2.02} 10^{-3}\right)$ & $1.09(454)$ \\
\hline Rev0045/301 & MOS1 (s7) & $9.27_{-3.27}^{+3.17} \quad 10^{20}$ & $0.74_{-0.02}^{+0.02}$ & $\left(8.93_{-1.13}^{+1.22}\right)$ & $2.28_{-0.14}^{+0.12}$ & $\left(\begin{array}{ll}8.65_{-1.71}^{+1.66} & 10^{-3}\end{array}\right)$ & $1.02(271)$ \\
\hline Rev0066/101 & $\mathrm{PN}$ & $0.40_{-0.40}^{+3.34} \quad 10^{20}$ & & & $1.89_{-0.06}^{+0.10}$ & $1.85_{-0.07}^{+0.14} 10^{-4}$ & $1.08(54)$ \\
\hline \multirow[t]{2}{*}{ Rev0092/201 } & $\mathrm{PN}$ & $\begin{array}{ll}6.19_{-0.47}^{+0.55} & 10^{20}\end{array}$ & $0.22_{-0.01}^{+0.01}$ & $766.2_{-62.0}^{+81.6}$ & $2.05_{-0.02}^{+0.02}$ & $9.16_{-0.20}^{+0.24} 10^{-3}$ & $1.15(1000)$ \\
\hline & MOS1 (s7) & $3.07_{-0.90}^{+0.91} \quad 10^{20}$ & $0.26_{-0.01}^{+0.01}$ & $380.1_{-50.1}^{+58.3}$ & $1.97_{-0.04}^{+0.03}$ & $1.11_{-0.05}^{+0.05} 10^{-2}$ & $1.49(376)$ \\
\hline
\end{tabular}

${ }^{a}$ The Galactic line-of-sight absorption $\left(=3.210^{20} \mathrm{~cm}^{-2}\right)$ has been subtracted. We assumed a metallicity of 0.4 times the solar value, i.e., $Z=0.008$.

Table 4. Fits to the RGS data: absorbed disk blackbody and power law

\begin{tabular}{|c|c|c|c|c|c|c|c|}
\hline Observation $^{a}$ & & $n_{\mathrm{H}}^{b}$ & $T_{\text {in }}$ & $A_{\text {disk }}$ & $\Gamma^{c}$ & $A_{\mathrm{pl}}$ & $\chi_{\nu}^{2}(\mathrm{dof})$ \\
\hline Rev0028/201 & RGS2 (s1) & $4.37_{-0.35}^{+1.37} \quad 10^{20}$ & $0.55_{-0.02}^{+0.02}$ & $183.8_{-15.6}^{+17.1}$ & 2.7 & $\begin{array}{ll}0.00_{-0.00}^{+0.25} & 10^{-2}\end{array}$ & $1.38(485)$ \\
\hline \multirow[t]{2}{*}{ Rev0045/101 } & RGS1 (s1) & $8.30_{-2.85}^{+2.73} \quad 10^{20}$ & $0.63_{-0.06}^{+0.07}$ & $60.73_{-22.18}^{+25.47}$ & 2.5 & $1.10_{-0.58}^{+0.65} 10^{-2}$ & $1.04(545)$ \\
\hline & RGS2 (s1) & $\begin{array}{lll}5.04_{-1.07}^{+3.67} & 10^{20}\end{array}$ & $0.49_{-0.03}^{+0.03}$ & $195.0_{-38.4}^{+5.8}$ & 2.5 & $0.55_{-0.55}^{+7.92} \quad 10^{-3}$ & $1.14(488)$ \\
\hline \multirow[t]{2}{*}{ Rev0045/201 } & RGS1 (s1) & $8.13_{-2.78}^{+2.63} 10^{20}$ & $0.67_{-0.06}^{+0.08}$ & $51.16_{-19.01}^{+22.14}$ & 2.5 & $1.06_{-0.56}^{+0.62} 10^{-2}$ & $1.08(543)$ \\
\hline & RGS2 (s1) & $\begin{array}{ll}7.57_{-2.92}^{+3.71} & 10^{20}\end{array}$ & $0.50_{-0.03}^{+0.03}$ & $178.5_{-40.9}^{+23.7}$ & 2.5 & $4.32_{-4.32}^{+9.3} 10^{-3}$ & $1.05(484)$ \\
\hline \multirow[t]{4}{*}{ Rev0045/301 } & RGS1 (s1) & $5.20_{-1.38}^{+4.76} \quad 10^{20}$ & $0.53_{-0.05}^{+0.05}$ & $127.4_{-41.7}^{+54.8}$ & 2.5 & $0.45_{-0.45}^{+9.13} 10^{-3}$ & $1.12(543)$ \\
\hline & RGS1 (s3) & $10.05_{-4.28}^{+3.96} 10^{20}$ & $0.70_{-0.10}^{+0.18}$ & $42.99_{-24.12}^{+14.98}$ & 2.5 & $1.07_{-0.81}^{+0.96} 10^{-2}$ & $0.91(541)$ \\
\hline & RGS1 (s5) & $7.45_{-2.24}^{+4.04} 10^{20}$ & $0.53_{-0.04}^{+0.06}$ & $119.0_{-38.5}^{+53.3}$ & 2.5 & $0.82_{-0.82}^{+7.84} \quad 10^{-3}$ & $1.08(540)$ \\
\hline & RGS2 (s4) & $9.90_{-2.73}^{+5.18} \quad 10^{20}$ & $0.45_{-0.03}^{+0.03}$ & $252.7_{-74.7}^{+91.0}$ & 2.5 & $3.18_{-3.18}^{+13.5} 10^{-3}$ & $1.03(487)$ \\
\hline Rev0066/101 & RGS1 (s7) & $\begin{array}{ll}0.00_{-0.00}^{+7.77} & 10^{20}\end{array}$ & $0.07_{-0.03}^{+0.03}$ & $3311_{-3056}^{+4171}$ & 1.9 & $2.77_{-0.20}^{+0.20} 10^{-4}$ & $1.11(44)$ \\
\hline \multirow[t]{2}{*}{ Rev0092/101 } & RGS1 (s2) & $5.85_{-1.06}^{+1.03} 10^{20}$ & $0.33_{-0.02}^{+0.01}$ & $211.2_{-25.3}^{+29.6}$ & 2.05 & $0.93_{-0.25}^{+0.26} 10^{-2}$ & $1.27(538)$ \\
\hline & RGS2 (s2) & $8.01_{-1.12}^{+0.95} 10^{20}$ & $0.27_{-0.02}^{+0.02}$ & $478.1_{-81.9}^{+103.8}$ & 2.05 & $1.36_{-0.14}^{+0.28} 10^{-2}$ & $1.25(485)$ \\
\hline \multirow[t]{2}{*}{ Rev0092/201 } & RGS1 (s4) & $6.40_{-1.45}^{+1.35} \quad 10^{20}$ & $0.29_{-0.03}^{+0.03}$ & $252.0_{-52.9}^{+83.9}$ & 2.05 & $1.06_{-0.31}^{+0.28} 10^{-2}$ & $1.08(537)$ \\
\hline & RGS2 (s5) & $6.84_{-1.28}^{+1.19} 10^{20}$ & $0.27_{-0.03}^{+0.03}$ & $500.0_{-52.7}^{+65.1}$ & 2.05 & $0.99_{-0.10}^{+0.09} \quad 10^{-2}$ & $1.15(485)$ \\
\hline
\end{tabular}

${ }^{a}$ We do not show fit parameters for observations without enough counts or without a reliable energy calibration. We assumed a metallicity of 0.4 times the solar value, i.e., $Z=0.008$.

${ }^{b}$ The Galactic line-of-sight absorption $\left(=3.210^{20} \mathrm{~cm}^{-2}\right)$ has been subtracted.

${ }^{c}$ Fixed.

the EPIC fits (with a correction to the value of $\Gamma$ from Rev0045 to take the pile-up into account). Because of the low count rate, the data in the Rev0066 observations are binned in groups of 100 channels each. The error-weighted means of the fit values of $T_{\text {in }}$ are $0.52 \mathrm{keV}$ and $0.29 \mathrm{keV}$ for observations before and after Rev0066 respectively.

We do not find strong evidence of emission lines. The column density $n_{\mathrm{H}}$ was generally below $10^{21} \mathrm{~cm}^{-2}$, except for Rev0066, where $n_{\mathrm{H}}$ is not well constrained. After the line-of-sight column density to the LMC is subtracted, the value of the column density for the observations before Rev0066 is $(5.50 \pm 0.67) 10^{20} \mathrm{~cm}^{-2}$, and the value after Rev0066 is $(6.83 \pm 0.58) 10^{20} \mathrm{~cm}^{-2}$. (These values are smaller than those obtained from the fits to the EPIC data.) Given that the error may be dominated by systematic effects, the error obtained in the fits may not truly represent the statistical weights of the fit parameters. Apart from the conclusion that the spectra do not show 


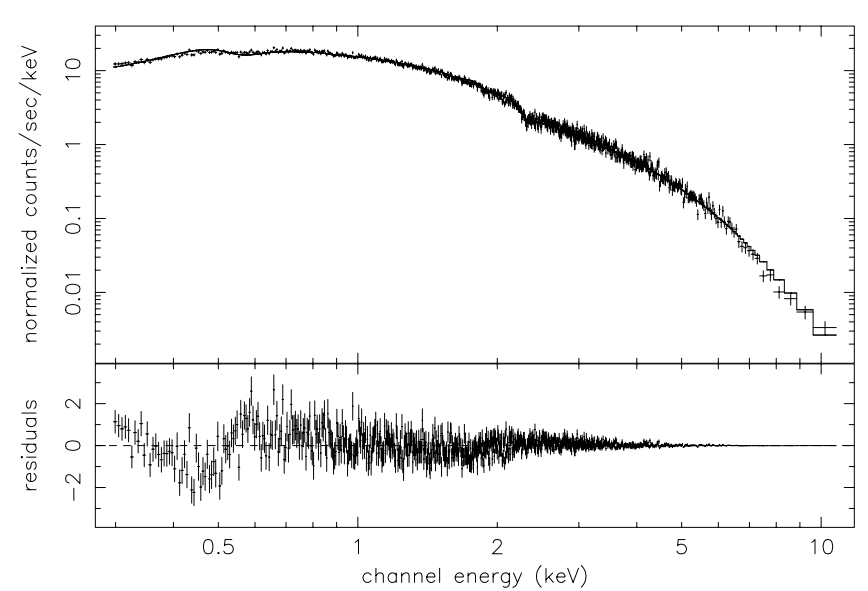

Fig. 2. The top panel shows the data and fit EPIC-PN spectra of the Rev0028/201 observation (2000 February 2). An absorbed diskbb+powerlaw model is used in the fit (see Table 3). The residuals are shown in the bottom panel

strong column absorption, we are unable to determine whether the variations in $n_{\mathrm{H}}$ are correlated with the X-ray luminosity.

\section{Discussion}

\subsection{Soft and hard states}

Previous RXTE/PCA observations (Wilms et al. 2000) have shown that when the source was bright (with $R X T E / A S M$ count rates $\gtrsim 3$ ct $\mathrm{s}^{-1}$ ), the disk blackbody component was prominent, and its fit temperature $T_{\mathrm{in}} \approx 1.0 \mathrm{keV}$. There was also a power-law tail with $\Gamma \approx 4$ in the spectra. The temperature $T_{\text {in }}$ appeared to decrease when the X-ray luminosity decreased, while the normalisation parameter $A_{\text {disk }}$ remained approximately constant. When the $R X T E / \mathrm{ASM}$ count rate dropped below $\approx 0.6$ ct s$^{-1}, T_{\text {in }}$ was reduced to $\approx 0.7 \mathrm{keV}$. The transition was accompanied by the hardening of the power-law component. The photon index $\Gamma$ became $\sim 2.0-3.0$. When the $R X T E / A S M$ count rate decreased below the 0.3 -ct $\mathrm{s}^{-1}$ level, the disk blackbody component was not detected, and the spectrum was a power law with $\Gamma=1.8$.

The soft-to-hard transition was also seen in our data. The spectra obtained in the Rev0028-Rev0045 observations show a disk blackbody component with $T_{\text {in }} \sim$ 0.6-1.0, and a power-law component with $\Gamma \approx 2.5$. The $R X T E / A S M$ count rate was below 2 ct $\mathrm{s}^{-1}$ during the observations. The spectral properties are similar to those observed previously when the system had similar $R X T E /$ ASM count rates.

The spectrum obtained in the Rev0066/101 observations is dominated by a power law with $\Gamma=1.9 \pm 0.1$. The photon index is consistent with that observed in the previous hard states (Wilms et al. 2000), while a lower value of $1.60 \pm 0.04$ was obtained by Boyd et al. (2000) on 2000 May 7. The $\chi^{2}$ of the fit which includes a thermal disk black-body component (best fit $T_{\text {in }}=0.14 \mathrm{keV}$ ) is

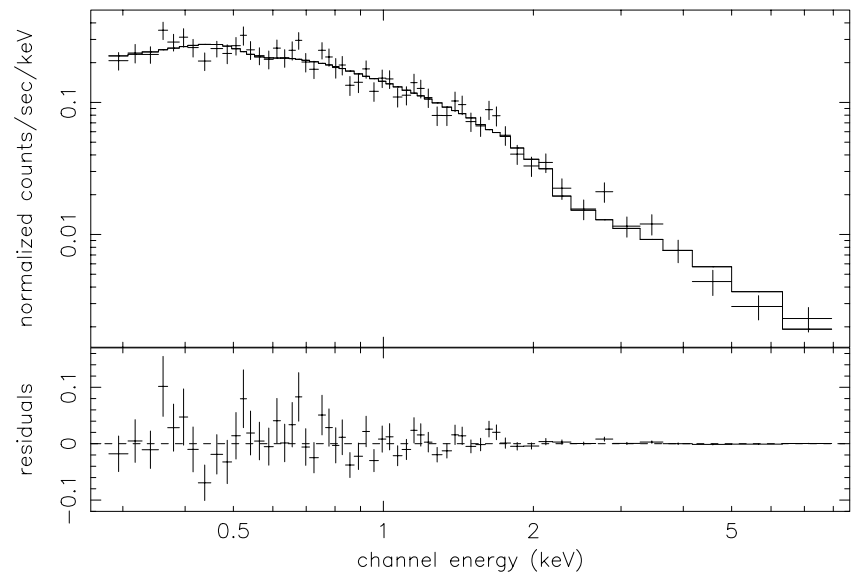

Fig. 3. Same as Fig. 2 for the Rev0066/101 EPIC-PN observation (2000 April 19)

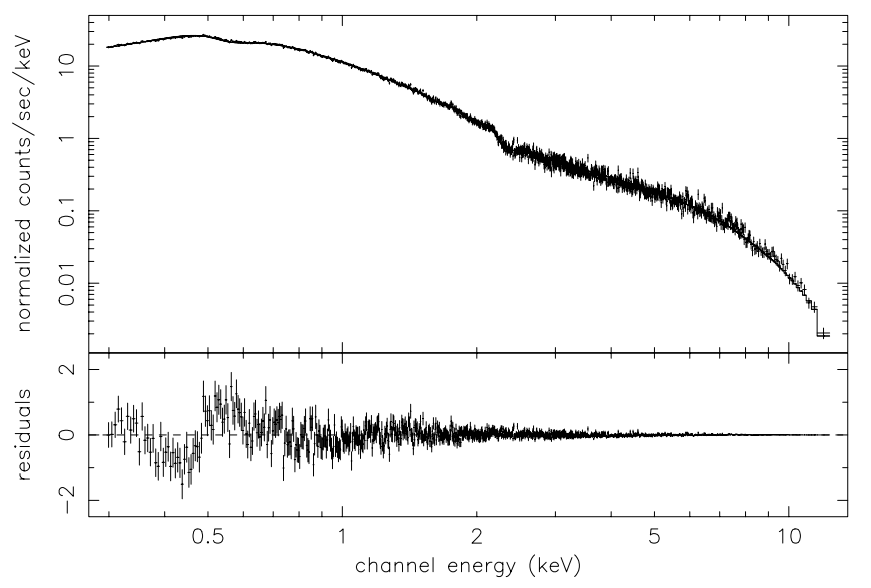

Fig. 4. Same as Fig. 2 for the Rev0092/201 EPIC-PN observation (2000 June 10)

58.486 for 52 degrees of freedom. If the thermal component is not included, then we obtain $\chi^{2}=58.493$ for 54 degrees of freedom. The thermal component is therefore insignificant.

The system was at the transition from the hard state back to the soft state during the Rev0092 observations (see Fig. 1). The power-law component had steepened, with $\Gamma=2.05 \pm 0.02$ (for the PN data). The disk blackbody component reappeared, with a temperature $T_{\mathrm{in}} \approx 0.2 \mathrm{keV}$, significantly lower than that before Rev0066.

In summary, between 2000 February and June LMC X-3 underwent a transition from a soft to a hard state, and then in the process of returning to the soft state. During the soft-to-hard transition the fit temperature of the disk blackbody component decreased, and the power law component became harder. As the system started to return to the soft state, the disk blackbody component became more prominent. The power law appeared to be steeper than that obtained from the RXTE/PCA observations near the middle of the faint state, yet the disk 
blackbody temperature was still well below the values of the previous soft state.

\subsection{Mode of mass transfer}

The RGS data constrain the value of $n_{\mathrm{H}}$ within the system to be $\lesssim 10^{21} \mathrm{~cm}^{-2}$, in contrast to the larger intrinsic column density expected for a companion with a strong stellar wind. The non-detection of obvious emission lines in the RGS spectra also indicates the absence of wind matter ejected in previous epochs (cf. the P Cygni lines seen in Cir X-1, Brandt \& Schulz 2000). Thus, the XMM-Newton spectral data do not support the mass-transfer scenario for LMC X-3 in which the black hole accretes matter mainly from a strong stellar wind from a massive companion. The high luminosity observed in the X-ray bands therefore requires the companion to overflow its Roche lobe.

A similar conclusion is also obtained independently from an analysis of the optical/UV properties of the system (Soria et al. 2001). The OM data obtained in the Rev0066 suggest that the companion is a B5 subgiant instead of a B3 main-sequence star. Roughly $3 \%$ of the $\mathrm{X}$-rays would be intercepted by the companion, so that the rate at which energy is deposited into its atmosphere can be $\gtrsim 510^{36} \mathrm{erg} \mathrm{s}^{-1}$ in the soft state. This rate is larger than the intrinsic luminosity of the companion. If the companion in LMC X-3 is indeed a subgiant star its tenuous envelope is susceptible to irradiation heating. The soft-to-hard transitions seen in the RXTE observations in 1997/1998 and the XMM-Newton observations in 2000 may be caused by variations in the rate of mass overflow from the Roche lobe of the subgiant companion.

Wilms et al. (2000)'s interpretation of the decreases in the RXTE/ASM count rate as evidence for transitions from soft to hard state is consistent with our data. We further propose that the decrease in the X-ray luminosity is caused by the decrease in the fraction of the Roche lobe filled by the companion star. When the companion is detached from its critical Roche surface, mass transfer will be dominated by a focused wind.

It is worth noting that the three known high-mass BHCs are all persistent X-ray sources which show preferential X-ray spectral states. While Cyg X-1 tends to be in the hard state, LMC X-1 and LMC X-3 are more often found in the soft state. Recent studies (e.g. Igumenshchev et al. 1999; Beloborodov \& Illarionov 2000) have shown that accretion of matter with low angular momentum will give rise to hard X-rays instead of soft X-rays. The relative angular momentum of the accreting matter is smaller for wind accretion than for Roche-lobe overflow. Cyg X-1 has a 33- $M_{\odot}$ O-type companion (Giles \& Bolton 1986), which has a strong stellar wind. The companion stars in LMC $\mathrm{X}-1$ and LMC X-3 are less massive $\left(\lesssim 10 M_{\odot}\right)$ B stars, whose stellar wind is much weaker. Therefore, we suggest a unified scenario which relates the mode of mass transfer (wind or Roche-lobe overflow) to the spectral state preferentially observed in these three high-mass BHC binaries (hard or soft respectively). The subgiant companion of LMC X-3 may occasionally underfill its Roche lobe because of feedback irradiative processes or instabilities in its envelope. This leads to the residual accretion of the (focused) wind matter which has relatively low angular momentum.

\section{Summary}

The BHC LMC X-3 was observed in 2000 February-June, with the XMM-Newton EPIC and RGS, throughout a softhard transition. The system was apparently in the process of returning to the soft state in 2000 June. The hard-state spectra are dominated by a power-law component with a photon index $\Gamma \approx 1.9$. The soft-state spectra consist of a thermal component with an inner-disk temperature $T_{\text {in }}$ of $\approx 0.9 \mathrm{keV}$ and a power-law tail with $\Gamma \approx 2.5-2.7$. The line-of-sight absorption deduced from the EPIC and RGS data is $n_{\mathrm{H}} \lesssim 10^{21} \mathrm{~cm}^{-2}$. Our observations therefore do not support the wind accretion model for this system in the soft state. The transition from the soft to the hard state appears to be a smooth process associated with the changes in the mass-transfer rate.

Acknowledgements. This work is based on observations obtained with XMM-Newton, an ESA science mission with instruments and contributions directly funded by ESA member states and the USA (NASA). We thank Keith Mason for his comments. KW acknowledges a PPARC visiting fellowship.

\section{References}

Beloborodov, A. M., \& Illarionov, A. 2000 [astro-ph/0006351] Boyd, P. T., \& Smale, A. P. 2000, IAUC, 7424

Boyd, P. T., Smale, A. P., Homan, J., et al. 2000, ApJ, 542, L127

Brandt, W. N., \& Schulz, N. S. 2000 [astro-ph/0007402]

Brinkman, A. C., Behar, E., Güdel, M., et al. 2001, A\&A, 365, L324

Caputo, M., Marconi, G., \& Ripepi, V. 1999, ApJ, 525, 784

Cowley, A. P., Crampton, D., Hutching, J. B., Remillard, R., \& Penfold, J. E. 1983, ApJ, 272, 118

Ebisawa, K., et al. 1993, ApJ, 403, 684

Giles, D. R., \& Bolton, C. T. 1986, ApJ, 304, 371

Igumenshchev, I. G., Illarionov, A. F., \& Abramowicz, M. A. 1999, ApJ, 517, L55

Leong, C., Kellogg, E., Gursky, H., Tanabaum, H., \& Giaccon, R. 1971, ApJ, 170, L67

Mazeh, T., van Paradijs, J., van den Heuvel, E. P. J., \& Savonije, G. J. 1986, A\&A, 157, 113

Mason, K. O., Breeveld, A., Much, R., et al. 2001, A\&A, 365, L36

Paczynski, B. 1983, ApJ, 273, L81

Paul, B., Kitamoto, S., \& Makino, F. 2000, ApJ, 528, 410

Soria, R., Wu, K., Page, M. J., \& Sakelliou, I. 2001, A\&A, 365, $\mathrm{L} 273$

Sunyaev, R. A., \& Titarchuk, L. G. 1980, A\&A, 86, 121

Titarchuk, L. G., \& Zannias, T. 1998, ApJ, 193, 863

Turner, M. J. L., Abbey, A., Arnaud, M., et al. 2001, A\&A, $365, \mathrm{~L} 27$

van Paradijs, J., et al. 1987, A\&A, 104, 201

Wilms, J., Nowak, M. A., Pottschmidt, K., et al. 2000 [astro-ph/0005489] 